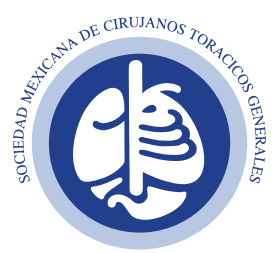

Vol. 1, Núm. 1

Enero-Abril 2020

pp 23-26

\title{
Sonda torácica, revisión de complicaciones en colocación y manejo por residentes de cirugía general ${ }^{\ddagger}$
}

\author{
Thoracic catheter, review of complications in placement \\ and management by general surgery residents
}

\author{
Edson René Marcos-Ramírez, * Alejandra Téllez-Aguilera," \\ Aracely Alejandra Guerrero-Arroyo," Manuel Wong-Jaén\$
}

Palabras clave:

Complicaciones, sonda, residente, entrenamiento, trauma.

Keywords:

Complications, catheter, resident, training, trauma.

\footnotetext{
* Residente de tercer año de Cirugía General; "Estudiante de quinto año de la carrera; $\S$ Médico adscrito, Cirujano de Tórax. Servicio de Cirugía General, Hospital Universitario "Dr. José Eleuterio González", UANL. Nuevo León, Monterrey, México.

‡ Presentado en el VII Congreso Internacional de la Sociedad Mexicana de Cirujanos Torácicos Generales en la Ciudad de Monterrey Nuevo León.
}

Recibido: 04/02/2019

Aceptado: 21/03/2019

Correspondencia: Edson R Marcos-Ramírez E-mail: ermarcos7@gmail.com

\begin{abstract}
RESUMEN
La colocación de una sonda torácica es un evento cotidiano en el trabajo de un cirujano, ya sea general o torácico, que muchas veces vemos como un procedimiento sencillo, por esto mismo la responsabilidad de realizar su colocación recae en el residente de menor grado, minimizando el hecho de que la colocación de una sonda torácica es un procedimiento invasivo a una cavidad de vital importancia y que el error conduce a una serie de eventos que perjudican al paciente. Esta práctica conlleva muchas veces que quien realiza la colocación tenga menos experiencia y resulte en mayores complicaciones, las cuales se aproximan al 20\% en diversas series y que van desde una colocación disfuncional sin lesión de estructuras intratorácicas hasta catastróficas como lesiones bronquiales, vasculares, hepáticas y esplénicas que ocasionan la necesidad de tratamientos quirúrgicos que no requería el paciente de manera inicial, aumentando la morbilidad y mortalidad. Los hospitales donde se entrenan residentes quirúrgicos deben tener presente esta realidad y considerar cambiar las prácticas para evitar estos problemas. Es por esto que se presenta la experiencia en complicaciones al colocar una sonda torácica en un centro de referencia de trauma en nuestro país.
\end{abstract}

\section{ABSTRACT}

Thoracic catheter placement is an everyday event in the work of a surgeon, whether general or thoracic, which we often see as a simple procedure, so the responsibility for placement lies with the resident of lesser degree, minimizing the fact that the placement of a chest probe is an invasive procedure into a cavity of vital importance and that the error leads to a series of events that harm the patient. These complications range from dysfunctional placement without injury to intra-thoracic structures, to catastrophic injuries such as bronchial, vascular, hepatic and splenic lesions that lead to the need for surgical treatments that the patient did not initially require, increasing morbidity and mortality. Hospitals where surgical residents are trained should be mindful of this reality and consider changing practices to avoid these problems. It is for this reason that we present the experience in complications when placing a thoracic catheter in a trauma reference center in our country.

\section{INTRODUCCIÓN}

La colocación de sonda torácica (ST) es un procedimiento antiguo descrito desde tiempos de Hipócrates que ha evolucionado de la mano de las artes quirúrgicas, siendo el principio básico el mismo, debe ser dominado por el médico general, ya que las especialidades quirúrgicas

Citar como: Marcos-Ramírez ER, Téllez-Aguilera A, Guerrero-Arroyo AA, Wong-Jaén M. Sonda torácica, revisión de complicaciones en colocación y manejo por residentes de cirugía general. Rev Mex Cir Torac Gen. 2020; 1(1):23-26 
son las expertas en el tema. ${ }^{1-3}$ Hoy en día es habitual el manejo de una ST por quienes tratan pacientes con patología torácica quirúrgica, misma que se realiza ya sea junto a la cama del paciente, en una sala de resucitación o un quirófano. Especialmente en pacientes con trauma torácico es de suma importancia evacuar el contenido pleural, previniendo las catastróficas consecuencias de un neumotórax a tensión o un hemotórax exanguinante. Es por lo antes mencionado, que el residente en entrenamiento debe dominar la técnica quirúrgica junto a la anatomía relacionada, las indicaciones y conocer las posibles complicaciones. Por este motivo, se presenta la experiencia de los residentes de cirugía general al abordar estos casos en el Hospital Universitario "Dr. José Eleuterio González" en Monterrey, N.L., centro de referencia de pacientes con trauma torácico.

\section{MATERIAL Y MÉTODOS}

Se trata de un estudio retrospectivo en el que incluimos 191 pacientes con trauma torácico tanto penetrante como contuso con necesidad de ST urgente o durante la evolución intrahospitalaria entre agosto de 2016 y agosto de 2018, estos procedimientos fueron realizados por residentes de cirugía general desde $1^{\text {er }}$ año hasta 5o año. Se obtuvo la información de expedientes clínicos, valorándose la estancia hospitalaria, así como las complicaciones derivadas de la colocación de la ST desde la recolocación por sonda disfuncional hasta la intervención quirúrgica mayor. Se obtuvo un total de 191 pacientes y se realizó un análisis estadístico con el programa JMP 10.

\section{RESULTADOS}

Nuestra serie constó de 191 pacientes con diagnóstico de trauma torácico y necesidad de colocación de ST en un lapso de dos años, de los cuales el 73.3\% (140 pacientes) fueron masculinos con una media de edad de 44 años. El 65.4\% de los casos fue trauma torácico penetrante (TTP), mientras que el $34.5 \%$ por trauma torácico contuso (TTC); se describen las características de la serie en el tabla 1. La entidad clínica más frecuente fue neumotórax con 80.1\% (153 pacientes del total), siendo secundario con mayor frecuencia en TTP, en tanto que los casos de hemotórax se obtuvieron de un total de 38 pacientes (19.9\%). La responsabilidad de la colocación de ST en nuestro hospital recae en residentes de los primeros tres años de la especialidad, siendo el 2 a año de residencia el de mayor número de sondas colocadas (110 ST colocadas, el 57.9\%) (Figura 1). En nuestro grupo de ST colocadas se observó que las complicaciones fueron menores en un $14.6 \%$ de los casos (28 pacientes). La subóptima colocación de la sonda con disfuncionalidad secundaria y la necesidad de recolocación se detectó en 25 de los 28 pacientes (89.2\%), el resto de las complicaciones fueron de casos aislados, por ejemplo, dos casos con fístula biliopleural (Tabla 2). De nuestra serie de pacientes, 52 de ellos (27.2\%) requirieron tratamiento quirúrgico mayor por toracotomía debido a la lesión inicial; sin embargo, se presentaron cuatro casos (7.6\%) en los que debido a la colocación de la sonda torácica fue necesario pasar a toracotomía: dos casos con fístula biliopleural, un caso con fístula broncopleural por la colocación de ST que

Tabla 1: Características de la población ( $N=191)$

$\begin{array}{lcc} & \mathrm{n} & \% \\ \text { Género } & & \\ \text { Masculino } & 140 & 73.30 \\ \text { Femenino } & 51 & 26.70 \\ \text { Edad (años) } & 46 & \\ \text { Etiología } & 125 & 65.45 \\ \text { TTP } & 66 & 34.55 \\ \text { TTC } & & 80.10 \\ \text { Diagnósticos } & 153 & 19.90 \\ \text { Neumotórax } & 38 & \end{array}$


Tabla 2: Complicaciones de sonda torácica (ST).

\begin{tabular}{lcc} 
Complicaciones & $\mathbf{n}$ & $\%$ \\
\hline Complicación en ST & 28 & 14.66 \\
Mala colocación & 25 & 89.29 \\
Fístula biliopleural & 2 & 7.14 \\
Lesión de arteria intercostal & 1 & 3.57 \\
Necesidad de cirugía & 4 & 7.69
\end{tabular}

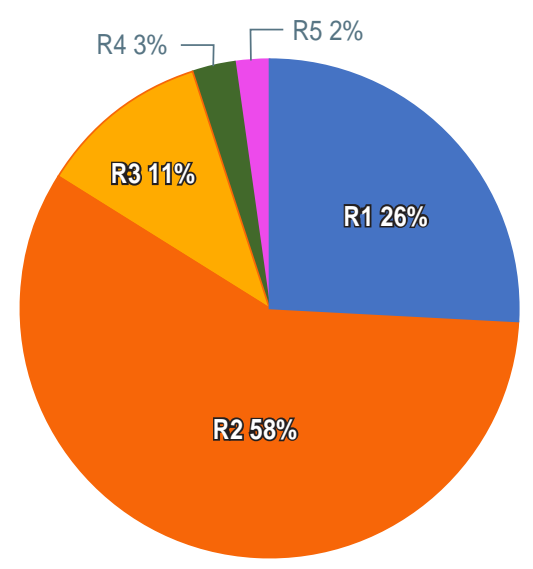

Figura 1: Frecuencia (\%) de los procedimientos realizados según el grado de residente.

requirió de reparación quirúrgica y un caso con lesión de arteria intercostal, el cual culminó en toracotomía con hemostasia. La mortalidad global de estos pacientes fue del 14.6\% (28 casos) todos ellos debido a la naturaleza de su traumatismo; no hubo defunciones atribuidas por alguna complicación derivada de la colocación de una ST. Los días de estancia intrahospitalaria (DEIH) son un punto importante a seguir, con una media de 9.4 días en esta casuística; sin embargo, en pacientes en quienes obtuvimos una complicación debida a la colocación de ST, la media de DIEIH aumentó a un 12.3 estadísticamente significativo $(p=0.03)$. No hubo diferencia estadística si se comparan los DEIH entre los diferentes grados del residente como responsable de la colocación de ST.

\section{DISCUSIÓN}

La responsabilidad de la colocación de ST habitualmente recae en residentes de especialidades quirúrgicas cursando sus primeros años. ${ }^{4}$ En especial, en nuestro país, son los residentes de cirugía general los que están expuestos, y quienes cuentan con menos experiencia, por lo que se espera que haya complicaciones. Sin embargo, este habitual procedimiento está asociado a una morbilidad significativa y ocasionalmente a mortalidad. ${ }^{4-6}$ Entre las complicaciones derivadas de este procedimiento en la literatura se reportan lesiones hepáticas, esplénicas por sondas colocadas intraabdominales, laceraciones pulmonares, lesiones en arteria intercostal como lo observamos en nuestro estudio, entre otros. ${ }^{4,7,8}$ Nuestra frecuencia de complicaciones por colocación de ST fue en un $14.6 \%$, similar a la descrita en la literatura. ${ }^{1,4,6}$ En una serie reportada por Bailey et al. en un hospital universitario en el Reino Unido se encontró que el $30 \%$ de sus sondas colocadas por residentes se complican con disfuncionalidad como la primera causa debido a sondas dobladas. ${ }^{6}$ Es interesante observar sus resultados con respecto a los nuestros. En otro estudio realizado por Chad et al. en Nueva Zelanda se incluyeron 338 pacientes que requirieron colocación de ST por etiología traumática; reportándose un $22.4 \%$ de pacientes con complicaciones, siendo la de mayor frecuencia la posicional con necesidad de recolocación, al igual que en nuestro centro. ${ }^{4}$ En general, con una definición de complicación por colocación de ST similar entre diversas series en la literatura, los rangos de complicaciones van de 5 a 15\%, siendo la complicación por posición errónea de la sonda la más frecuente en un $11.8 \% .9,10$ Se espera este tipo de complicaciones debido a que quienes colocaron todas estas sondas son personal en entrenamiento. Es por esto que los residentes de menor grado deben tener mayor supervisión por parte del personal con más experiencia para reducir una serie de complicaciones que derivan en tiempo de estancia intrahospitalaria más prolongada, mayores costos y hasta evitar la necesidad de tratamiento quirúrgico que inicialmente no se requería por la patología de base. 


\section{CONCLUSIÓN}

La importancia de la supervisión es un acto de suma importancia en procedimientos que se realizan día a día y que debido a que se le considera un procedimiento menor, se le resta importancia. Este tipo de estudios nos hace enfatizar las ventajas de reducir las complicaciones para el paciente y de forma secundaria para el hospital. En nuestro hospital-escuela reforzamos estos conceptos con evidencia fundada en nuestra experiencia, y así modificar conductas que beneficien tanto a los residentes como al principal actor que es el paciente.

\section{AGRADECIMIENTOS}

Nuestro agradecimiento es hacia los pacientes, quienes son la razón de ser de nuestro trabajo.

\section{REFERENCIAS}

1. Sudduth $\mathrm{CL}$, Shinnick JK, Geng Z, McCracken CE, Clifton MS, Raval MV. Optimal surgical technique in spontaneous pneumothorax: a systematic review and meta-analysis. J Surg Res. 2017; 210: 32-46. doi: 10.1016/j.jss.2016.10.024.
2. Varela G, Jiménez MF, Novoa N. Portable chest drainage systems and outpatient chest tube management. Thorac Surg Clin. 2010; 20(3): 421-426. doi: 10.1016/j.thorsurg.2010.03.006.

3. Durai $R$, Hoque $H$, Davies TW. Managing a chest tube and drainage system. AORN J. 2010; 91(2): 275-283. doi: 10.1016/j. aorn.2009.09.026.

4. Ball CG, Lord J, Laupland KB et al. Chest tube complications: How well are we training our residents? Can J Surg. 2007; 50(6): 450-458. doi: 10.1016/B978-012088576-3/50033-2.

5. Deneuville M. Morbidity of percutaneous tube thoracostomy in trauma patients. Eur J Cardiothorac Surg. 2002; 22(5): 673-678.

6. Bailey RC. Complications of tube thoracostomy in trauma. Emerg Med J. 2000; 17 (2): 111-114. doi: 10.1136/emj.17.2.111.

7. Roberts ME, Neville E, Berrisford RG, Antunes G, Ali NJ. Management of a malignant pleural effusion: British Thoracic Society pleural disease guideline 2010. Thorax. 2010; 65(Suppl. 2). doi: 10.1136/ thx.2010.136994.

8. Stawicki SA, Kwiatt M, Tarbox A et al. Thoracostomy tubes: A comprehensive review of complications and related topics. Int J Crit Illn Inj Sci. 2014; 4(2): 142. doi: 10.4103/2229-5151.134182.

9. Zehtabchi S, Rios CL. Management of emergency department patients with primary spontaneous pneumothorax: needle aspiration or tube thoracostomy? An Emerg Med. 2008; 51(1). doi: 10.1016/j. annemergmed.2007.06.009.

10. Tait P, Waheed U, Bell S. Successful removal of malpositioned chest drain within the liver by embolization of the transhepatic track. Cardiovasc Intervent Radiol. 2009; 32(4): 825-827. doi: 10.1007/ s00270-008-9461-y.

Conflicto de intereses: Ninguno. 